\title{
Thiamethoxam exposure induces endoplasmic reticulum stress and affects ovarian function and oocyte development in mice
}

Yu Liu ${ }^{a}$, Quan-Kuo He ${ }^{\mathrm{a}}$, Zhi-Ran Xü ${ }^{\mathrm{b}}$, Chang-Long Xuc, Si-Cheng Zhao ${ }^{\mathrm{a}}$, Yu-Shen Luo $^{a}$, Xue Sun ${ }^{\mathrm{a}}$, Zhong-Quan $\mathrm{Qi}^{\mathrm{a} *}$ and Hai-Long Wang ${ }^{\mathrm{d} *}$

${ }^{a}$ Medical College, Guangxi University, Nanning, Guangxi 530004, People’s Republic of China

b Center Translational medicine research, Ruikang Hospital Affiliated to Guangxi University of Chinese Medicine, Nanning, Guangxi 530011, People's Republic of China

c Reproductive Medical Center of Nanning Second People's Hospital, Nanning, Guangxi 530031, People's Republic of China

d Department of Basic Medicine, School of Medicine, Xiamen University, Xiamen, Fujian 361102, People's Republic of China

*Correspondence to: Hai-Long WANG, Department of Basic Medicine, School of Medicine, Xiamen University, Xiamen, Fujian 361102, People's Republic of China;

E-mail: hailongwang@xmu.edu.cn

Or to Zhong-Quan QI, Medical College, Guangxi University, Nanning, Guangxi 530004, People’s Republic of China; E-mail: yxyyz@gxu.edu.cn 


\section{Detailed Data}

\section{- Thiamethoxam exposure decreased ovary coefficient and disrupted}

\section{follicle development}

(1) Body weight increase:

Day 15: Control: $26.07 \pm 0.53 \mathrm{~g}, \mathrm{TMX}(50 \mathrm{mg} / \mathrm{kg} /$ day $): 25.40 \pm 0.50 \mathrm{~g}(\mathrm{P}>0.05), \mathrm{TMX}$ (100 mg/kg/day): $25.20 \pm 0.35 \mathrm{~g}(\mathrm{P}>0.05), \mathrm{TMX}(250 \mathrm{mg} / \mathrm{kg} /$ day $): 24.60 \pm 0.62 \mathrm{~g}(\mathrm{P}$ $<0.05)$.

Day 18: (Control: $26.70 \pm 0.60 \mathrm{~g}$, TMX $(50 \mathrm{mg} / \mathrm{kg} / \mathrm{day}): 26.77 \pm 0.50 \mathrm{~g}(\mathrm{P}>0.05)$, TMX (100 mg/kg/day): $25.67 \pm 0.45 \mathrm{~g}(\mathrm{P}>0.05)$, TMX $(250 \mathrm{mg} / \mathrm{kg} /$ day $): 25.17 \pm 0.61$ $\mathrm{g}(\mathrm{P}<0.05)$.

Day 21: Control: $27.40 \pm 0.60$ g, TMX (50 mg/kg/day): $27.60 \pm 0.60 \mathrm{~g}(\mathrm{P}>0.05), \mathrm{TMX}$ (100 mg/kg/day): $26.23 \pm 0.37 \mathrm{~g}(\mathrm{P}>0.05), \mathrm{TMX}(250 \mathrm{mg} / \mathrm{kg} / \mathrm{day}): 25.93 \pm 0.71 \mathrm{~g}(\mathrm{P}$ $<0.05)$.

Day 24: Control: $28.33 \pm 0.87 \mathrm{~g}, \mathrm{TMX}(50 \mathrm{mg} / \mathrm{kg} /$ day $): 28.10 \pm 0.56 \mathrm{~g}(\mathrm{P}>0.05), \mathrm{TMX}$ (100 mg/kg/day): $27.07 \pm 0.30 \mathrm{~g}(\mathrm{P}>0.05), \mathrm{TMX}(250 \mathrm{mg} / \mathrm{kg} / \mathrm{day}): 26.47 \pm 0.84 \mathrm{~g}(\mathrm{P}$ $<0.05)$.

Day 28: Control: $28.93 \pm 0.96$ g, TMX (50 mg/kg/day): $29.20 \pm 0.45 \mathrm{~g}(\mathrm{P}>0.05), \mathrm{TMX}$ $(100 \mathrm{mg} / \mathrm{kg} /$ day $): 27.93 \pm 0.26 \mathrm{~g}(\mathrm{P}>0.05), \mathrm{TMX}(250 \mathrm{mg} / \mathrm{kg} /$ day $): 26.87 \pm 0.73 \mathrm{~g}(\mathrm{P}$ $<0.05)$.

(2) Weight of ovary:

Control: $2.51 \pm 0.17$ g. TMX $(50 \mathrm{mg} / \mathrm{kg} /$ day $): 2.63 \pm 0.29 \mathrm{~g}(\mathrm{P}>0.05), \mathrm{TMX}(100$ $\mathrm{mg} / \mathrm{kg} /$ day $): 2.30 \pm 0.21 \mathrm{~g}(\mathrm{P}>0.05), \operatorname{TMX}(250 \mathrm{mg} / \mathrm{kg} /$ day $): 1.97 \pm 0.19 \mathrm{~g}(\mathrm{P}<0.05)$. 


\section{- Thiamethoxam exposure disrupts the expression of female hormone receptor}

(1) Gray value quantified for FSHR: Control: $1.00 \pm 0.14$, thiamethoxam (50 $\mathrm{mg} / \mathrm{kg} /$ day $): 0.98 \pm 0.23(\mathrm{P}>0.05)$, thiamethoxam $(100 \mathrm{mg} / \mathrm{kg} /$ day $): 0.39 \pm 0.12(\mathrm{P}<$ $0.05)$, thiamethoxam $(250 \mathrm{mg} / \mathrm{kg} /$ day $): 0.46 \pm 0.14(\mathrm{P}<0.05)$

(2) Gray value quantified for AMHR: Control: $1.00 \pm 0.07$, thiamethoxam (50 $\mathrm{mg} / \mathrm{kg} /$ day $): 1.05 \pm 0.10(\mathrm{P}>0.05)$, thiamethoxam $(100 \mathrm{mg} / \mathrm{kg} /$ day $): 0.93 \pm 0.14(\mathrm{P}>$ 0.05); thiamethoxam $(250 \mathrm{mg} / \mathrm{kg} /$ day $): 0.54 \pm 0.19(\mathrm{P}<0.05)$

(3) Gray value quantified for ER- $\beta$ : Control: $1.00 \pm 0.10$, thiamethoxam $(50 \mathrm{mg} / \mathrm{kg} /$ day $)$ : $0.72 \pm 0.14(\mathrm{P}>0.05)$, thiamethoxam $(100 \mathrm{mg} / \mathrm{kg} / \mathrm{day}): 0.48 \pm 0.08(\mathrm{P}<0.05)$; thiamethoxam $(250 \mathrm{mg} / \mathrm{kg} /$ day $): 0.50 \pm 0.11(\mathrm{P}<0.05)$

(4) Gray value quantified for LHR: Control: $1.00 \pm 0.09$, thiamethoxam (50 mg/kg/day): $0.65 \pm 0.19(\mathrm{P}>0.05)$, thiamethoxam $(100 \mathrm{mg} / \mathrm{kg} /$ day $): 0.44 \pm 0.10(\mathrm{P}<0.05)$, thiamethoxam $(250 \mathrm{mg} / \mathrm{kg} /$ day $): 0.09 \pm 0.04(\mathrm{P}<0.05)$

\section{- Thiamethoxam exposure induces apoptosis in ovarian granulosa cells}

(1) Ration of TUNEL positive follicle: Control: $15.33 \pm 4.33$, TMX (100 mg/kg/day): $32.67 \pm 6.36(\mathrm{P}>0.05)$, TMX $(250 \mathrm{mg} / \mathrm{kg} /$ day $): 50.67 \pm 5.70(\mathrm{P}<0.05)$

(2) Gray value quantified for Cleaved-Caspase 3: Control: $1.00 \pm 0.17$, thiamethoxam $(50 \mathrm{mg} / \mathrm{kg} /$ day $): 1.92 \pm 0.29(\mathrm{P}<0.05)$, thiamethoxam $(100 \mathrm{mg} / \mathrm{kg} /$ day $): 3.46 \pm 0.55(\mathrm{P}$ $<0.05)$, thiamethoxam $(250 \mathrm{mg} / \mathrm{kg} /$ day $): 4.75 \pm 0.43(\mathrm{P}<0.05)$

(3) Gray value quantified for Bax: Control: $1.00 \pm 0.08$, thiamethoxam (50 mg/kg/day): $1.48 \pm 0.20(\mathrm{P}<0.05)$, thiamethoxam $(100 \mathrm{mg} / \mathrm{kg} /$ day $): 1.59 \pm 0.21(\mathrm{P}<0.05)$, thiamethoxam $(250 \mathrm{mg} / \mathrm{kg} /$ day $): 2.06 \pm 0.17(\mathrm{P}<0.05)$

(4) Gray value quantified for Bcl2: Control: $1.00 \pm 0.04$, thiamethoxam (50 mg/kg/day): $0.51 \pm 0.10(\mathrm{P}<0.05)$, thiamethoxam $(100 \mathrm{mg} / \mathrm{kg} / \mathrm{day}): 0.41 \pm 0.06(\mathrm{P}<0.05)$, thiamethoxam $(250 \mathrm{mg} / \mathrm{kg} /$ day $): 0.44 \pm 0.08(\mathrm{P}<0.05)$ 


\section{- Thiamethoxam exposure impedes oocytes but not embryo}

\section{development}

(1) Number of collected oocytes: Control: $41.3 \pm 1.8$, thiamethoxam $(50 \mathrm{mg} / \mathrm{kg} /$ day): $39.0 \pm 3.8(\mathrm{P}>0.05)$, thiamethoxam $(100 \mathrm{mg} / \mathrm{kg} /$ day $): 27.0 \pm 3.2(\mathrm{P}<0.05)$, thiamethoxam $(250 \mathrm{mg} / \mathrm{kg} /$ day $): 22.7 \pm 3.3(\mathrm{P}<0.05)$

(2) GVBD rate: Control: $85.50 \pm 1.11(\%)$, thiamethoxam $(50 \mathrm{mg} / \mathrm{kg} /$ day $): 87.07 \pm$ $2.72(\%)(\mathrm{P}>0.05)$, thiamethoxam $(100 \mathrm{mg} / \mathrm{kg} / \mathrm{day}): 81.20 \pm 4.23(\%)(\mathrm{P}>0.05)$, thiamethoxam $(250 \mathrm{mg} / \mathrm{kg} /$ day $): 65.40 \pm 4.46(\%)(\mathrm{P}<0.05)$

(3) PB extrusion rate: Control: $77.30 \pm 2.36(\%)$, thiamethoxam $(50 \mathrm{mg} / \mathrm{kg} / \mathrm{day}): 75.73$ $\pm 3.54(\%)(\mathrm{P}>0.05)$, thiamethoxam $(100 \mathrm{mg} / \mathrm{kg} / \mathrm{day}): 64.43 \pm 4.97(\%)(\mathrm{P}>0.05)$, thiamethoxam (250 mg/kg/day): $52.77 \pm 6.70(\%)(\mathrm{P}<0.05)$

(4) Number of zygotes obtained: Control: $16.6 \pm 0.7$, thiamethoxam (50 $\mathrm{mg} / \mathrm{kg} /$ day $): 14.6 \pm 2.0(\mathrm{P}>0.05)$, thiamethoxam $(100 \mathrm{mg} / \mathrm{kg} /$ day $): 10.8 \pm 1.8(\mathrm{P}<0.05)$, thiamethoxam $(250 \mathrm{mg} / \mathrm{kg} /$ day $): 9.4 \pm 0.9(\mathrm{P}<0.05)$

\section{- Effects of Thiamethoxam on DNA damage and early apoptosis in}

\section{mouse oocytes}

(1) Number of $\gamma$-H2AX foci: Controls: $2.68 \pm 0.36$, thiamethoxam: $18.47 \pm 0.92, \mathrm{P}<$ 0.05

(2) Ratio of apoptotic oocytes: Control: $7.73 \pm 2.94$, thiamethoxam $(250 \mathrm{mg} / \mathrm{kg} /$ day $)$ : $29.97 \pm 5.10(\mathrm{P}<0.05)$ 
- Thiamethoxam exposure increases levels of reactive oxygen species and induces endoplasmic reticulum stress

(1) Fluorescence intensity of DCFH-DA: Control: $1.00 \pm 0.08$, thiamethoxam (250 $\mathrm{mg} / \mathrm{kg} /$ day $): 1.83 \pm 0.13(\mathrm{P}<0.05)$

(2) Expression of CAT: Control: 1.00, thiamethoxam (250 mg/kg/day): $1.94 \pm 0.24$ $(\mathrm{P}<0.05)$

(3) Expression of GSH-Px: Controls: 1.00, thiamethoxam: $1.64 \pm 0.08(\mathrm{P}<0.05)$

(4) Expression of SOD: Controls: 1.00, thiamethoxam: $0.95 \pm 0.22(\mathrm{P}>0.05)$

- Thiamethoxam exposure alters mitochondrial morphology and function

(1) Mitochondrial clusters: Control: $11.85 \pm 2.70$ (\%); thiamethoxam $(250 \mathrm{mg} / \mathrm{kg} /$ day):

$39.56 \pm 4.86(\%)(\mathrm{P}<0.05)$

(2) Red/Green Ratio of JC-1: Control: $0.67 \pm 0.05$, thiamethoxam $(250 \mathrm{mg} / \mathrm{kg} / \mathrm{day}): 1.53$

$\pm 0.10(\mathrm{P}<0.05)$ 